\title{
Geschlecht, Ethnie und öffentliche Moral: Migrantinnen in der holländischen Frauen- und Minoritätspolitik
}

\section{Einleitung}

Infolge großer Migrationsbewegungen sind heute die meisten europäischen Gesellschaften multikulturell. Dies hat der Frage, wie der Staat kulturelle Differenzen aufnehmen soll, bei den Politikern eine neue Dringlichkeit verschafft. Die öffentliche Diskussion über Multikulturalismus wurde allerdings bisher hauptsächlich in bezug auf die Rechte und Interessen kultureller Minderheiten geführt. Häufig wird vergessen, daß Minderheitenpopulationen sich nicht nur aus Männern, sondern aus Männern und Frauen zusammensetzen. ${ }^{1}$

Es ist zu erwarten, daß die Rechte und Interessen von Migrantinnen als Frauen sich nicht immer mit ihren Rechten als Angehörige einer Minderheit decken. ${ }^{2}$ Aufgrund des Mangels an Aufmerksamkeit für Migrantinnen wird dieses Thema jedoch kaum berücksichtigt. Dieser Artikel unternimmt am Beispiel der Niederlande einen Versuch, dieser Vernachlässigung etwas entgegenzusetzen. Er befaßt sich mit der Frage, ob in der holländischen Politik, die Frauen aus ethnischen Minderheiten betrifft, die Achtung vor kultureller Verschiedenheit (bezüglich der Werte) mit Werten, die sich auf die Emanzipation von Frauen beziehen, wie Autonomie und Gleichberechtigung, in Einklang steht oder nicht. Ich habe mich besonders für die Frauenpolitik bei ethnischen Minderheiten interessiert, weil ein hoher Prozentsatz der Immigranten in den Niederlanden türkischer oder marokkanischer Herkunft ist. Das bedeutet, sie haben einen islamischen Hintergrund. In der öffentlichen Meinung in den Niederlanden gilt der Islam als eine undemokratische Religion, der Frauen diskriminiert und unterdrückt. Meine Frage war, ob die ausführliche Kritik des Orientalismus von E.W. Said (1978), welche sich auf die westlichen Vorstellungen über den Orient richtete, sich auch auf diese sehr spezielle Politik anwenden ließe.

Dieser Artikel ist folgendermaßen aufgebaut. Er beginnt mit der Vorstellung von Saids Kritik des Orientalismus, von der für die vorliegende Analyse wich- 
tige Impulse ausgegangen sind. Anschließend wird etwas zur Immigration und zur sozialen Lage von Frauen aus verschiedenen Immigrantengruppen gesagt. Es folgt eine Diskussion der beiden politischen Strategien, die auf die Lage der Frauen aus ethnischen Minderheiten ausgerichtet ist, d.h. der Minderheitenpolitik und der Emanzipationspolitik. Anschließend werde ich mich damit auseinandersetzen, wie die Programme in die Praxis umgesetzt werden. Die jüngsten Entwicklungen werden aufgenommen und diskutiert; besonders in der Emanzipationspolitik scheint eine Verschiebung stattgefunden zu haben: auf kulturelle Verschiedenheit in ihrem Verhältnis zur Emanzipation wird jetzt stärker reflektiert. Der Aufsatz schließt ab mit einer Würdigung der holländischen Politik bezüglich ihrer Verknüpfung von kultureller Differenz und Frauenemanzipation.

\section{Orientalismus und Frauenemanzipation}

„Orientalismus“ ist zunächst der Titel eines Buches, in dem Edward Said seine kritische Rekonstruktion britischer, französischer und us-amerikanischer Forschung und Literatur über den Orient vorgestellt hat. Dabei beschränkt Said sich auf Darstellungen des Nahen Ostens seit Beginn des achtzehnten Jahrhunderts bis heute. ,"Orientalismus' bezieht sich auch auf einen bestimmten Denkstil, der sich in einer ontologischen und metaphysischen Unterscheidung zwischen dem ,Orient ' und - in den meisten Fällen - , dem Okzident ' begründet." (Said 1978, 2). In dieser okzidentalen Repräsentation des „Orients" ist der Westen rational, entwickelt, human und überlegen, während der Osten anomal, unterentwickelt und unterlegen ist. Innerhalb dieses Repräsentationssystems erscheint der Osten als zeitlos, gleichförmig und unfähig, sich selbst zu bestimmen. Vom Westen dagegen wird postuliert, er sei modern und dynamisch (Said 1978, 300f.). Said behauptet, daß der Orientalismus als Denkstil einen besonderen Stil von Herrschaft und Unterdrückung begründete. Viele europäische Kolonialisten hatten nämlich das Gefühl, ihre Kolonisierung diene einer Mission, sie brächten die Zivilisation. Natürlich waren sie bezüglich ökonomischer Interessen nicht blind, aber sie hielten doch gleichzeitig ihre Kolonialherrschaft für die Erfüllung der Aufgabe sogenannter zivilisierter Völker. In diesem Sinne war der Orientalismus keine Heuchelei oder bloßer Ethnozentrismus oder falsches Bewußtsein, sondern eine in sich kohärente Art des Denkens über eine geographische Lage, die man als „Orient" bezeichnete. Said beschäftigt sich mit der Stellung der Frau in der westlichen Repräsentation des Orients kaum. Feministische Wissenschaftlerinnen haben darauf hingewiesen, da $\beta$ der Islam als eine „orientalische“ Religion im Westen als barbarische Religion dargestellt wird, die Frauen unterdrückt, während muslimische Frauen als Leidende und passive Opfer dargestellt werden. In diesem Zusammenhang werden westliche Frauen grundsätzlich als bewußt und muslimische bzw. „orientalische“ Frauen als unbewußt behandelt. Leila Ahmed zum Beispiel wirft westlichen Feministinnen vor, daß sie völlig unkritisch die Maxime des Orientalismus übernähmen, Europa habe den 
Osten die Bedeutung der Freiheit zu lehren: „Sich vorzustellen, wir existierten gedankenlos, passiv, gleichgültig und seien uns möglicherweise unserer Unterdrückung nicht bewußt, wir ertruigen eine Situation, die keine westliche Frau ertragen würde (dabei ertragen viele in der Isolation der Kernfamilie wesentlich mehr), bedeutet, unsere „Inferiorität“ vorauszusetzen und zu implizieren." (Ahmed 1982; 530; vgl. auch Ahmed 1992, Kabbani 1986, Lewis 1996, Lutz 1991, Saadawi 1980). Das Erbe des Orientalismus führt in diesem Kontext dazu, daß Emanzipation nur bedeuten kann, die östlichen Werte aufzugeben und die westlichen zu übernehmen.

\section{Migrantinnen in den Niederlanden}

Der Anteil der Immigranten in den Niederlanden umfaßt etwa 7\% der Gesamtbevölkerung und setzt sich aus einer großen Vielfalt von Gruppen zusammen. Es kann zwischen Migranten aus den früheren holländischen Kolonien, Arbeitsmigranten und politischen Flüchtlingen unterschieden werden. Die drei größten Gruppen unter denjenigen, an die holländische Minderheitenpolitik sich wendet und auf die deren Zugriff sich auswirkt, sind dementsprechend die surinamesische Gruppe mit etwa 290000 Personen, die türkische Gruppe mit etwas 265000 und die marokkanische Gruppe, die ungefähr 225000 Personen umfaßt. In meiner Darstellung der Lage von Migrantinnen werde ich mich auf Frauen aus diesen drei größten Gruppen konzentrieren.

Die meisten Surinamesinnen kamen in den 1970ern in die Niederlande. Sie kamen aufgrund ökonomischer Motive und weil sie die Ungewißheit der Situation nach der Unabhängigkeit im Jahre 1975 fürchteten, in der sie nicht wußten, was sie erwartete. Seit 1960 hatte die niederländische Regierung Arbeitsmigranten aus der Türkei und Marokko als Hilfsarbeiter für ungelernte Tätigkeiten in den alten arbeitsintensiven Industriezweigen angeworben. Mit dem ökonomischen Rückgang hörte die Rekrutierung von Arbeitsmigranten seit 1973 auf. Dies galt allerdings nicht für die Einwanderung, da in den siebziger Jahren im Zuge der Familienzusammenführung immer mehr Arbeitsmigranten zuzogen. Die meisten türkischen und marokkanischen Frauen kamen ihren Ehemännern und Vätern in die Niederlande nach, um mit ihnen zusammenzuleben. Der Höhepunkt der Familienzusammenführung lag zwischen 1979 und 1981. So leben surinamesische, türkische und marokkanische Frauen nunmehr seit etlichen zwanzig Jahren in den Niederlanden.

Verglichen mit niederländischen Frauen sind die Frauen aus allen drei Gruppen im Rückstand, sowohl hinsichtlich Bildung und Ausbildung als auch bezüglich ihrer Erwerbsarbeitsposition. Zwar sind innerhalb der eigenen Gruppe die Mädchen im Bildungsbereich besser als die Jungen, aber im Vergleich zu holländischen Mädchen ist ihr Bildungsniveau immer noch wesentlich niedriger. Es läßt sich beobachten, daß unter den Erwerbsarbeit suchenden Frauen die Arbeitslosenzahlen vor allem bei den Marokkanerinnen in den vergangenen Jahren eine dramatische Höhe erreicht hatten. Gegenwärtig (1997) ist die Arbeitslosigkeit in dieser Gruppe etwas zurückgegangen und liegt nun etwa auf 
dem gleichen Niveau wie bei den türkischen Frauen, d.h. bei einer Erwerbslosenquote von $46 \%$. Das ist im Vergleich zu einer Erwerbslosenquote von 14\% bei den niederländischen Frauen immer noch sehr hoch. Surinamesische Frauen nehmen mit einer Erwerbslosenquote von 27\% eine mittlere Position ein. Die türkischen und marokkanischen Frauen, die einer Erwerbstätigkeit nachgehen, verrichten meistens ungelernte Tätigkeiten, dabei haben türkische Frauen eher einen Arbeitsplatz in der Industrie und marokkanische arbeiten als Büroreinigungskräfte. Surinamesische Frauen sind gleichmäßiger in allen Sektoren des Arbeitsmarktes vertreten und obwohl die meisten von ihnen niedrige oder ungelernte Arbeitstätigkeiten ausüben, verfügt eine Teil von ihnen doch über höhere Bildung und arbeitet in höheren Positionen.

Alles in allem kann die soziale Position von Frauen aus diesen Migrantengruppen als schwach gekennzeichnet werden. Die Besorgnis über ihre soziale Stellung war für die niederländische Regierung der Anlaß, ein politisches Programm zu entwickeln, das Frauen- und Minderheitenpolitik miteinander verbinden sollte. Diesem Programm werde ich mich jetzt zuwenden.

\section{4. „Kultur" in der Minderheiten- und in der Emanzipationspolitik}

Die beiden Memoranden, die den allgemeinen Rahmen für eine Frauen- und Minderheitenpolitik abstecken, sind das Minderheitenmemorandum von 1983 und der emanzipationspolitische Plan von 1985. Die Problemanalyse, die in diese Memoranden Eingang gefunden hat, dient immer noch als Grundlage für die politischen Strategien, die im Hinblick auf Migrantinnen verfolgt werden. Es haben sich allerdings im Laufe der Jahre in der Implementierung der politischen Programme einige Verschiebungen ergeben, bemerkenswerterweise betrifft das aber die Minderheitenpolitik stärker als die Emanzipationspolitik.

Die holländische Minderheitenpolitik zielt auf sieben Gruppen, darunter die Surinamesen, Türken und Marokkaner. Das Hauptziel der Minderheitenpolitik besteht darin, Gleichstellung und volle Chancengleichheit in bezug auf gesellschaftliche Partizipation anzubieten. 1983 wurde dieses Ziel auch in der Formulierung „Integration unter Aufrechterhaltung der kulturellen Identität“ ausgedrückt. Damit sollte deutlich gemacht werden, daß die niederländische Regierung die Auffassung vertritt, eine multikulturelle Gesellschaft könne nur dann gut funktionieren, wenn die Kulturen der verschiedenen ethnischen Minderheiten gleich behandelt werden, und da $\beta$ die Regierung daher nicht auf Assimilation abziele. Dieses Prinzip kultureller Gleichheit wurde in den letzten Jahren in Maßnahmen umgesetzt, die unter ethnischen Minderheiten einen Anreiz zur Selbstorganisation schaffen sollten und in Förderungsmaßnahmen, mit denen die Gruppen in ihrer Religionsausübung unterstützt wurden. Unter dem selben Vorzeichen standen auch Maßnahmen zur interkulturellen Erziehung. Bereits in einem recht frühen Stadium stellte sich heraus, daß die politische Praxis wesentlich mehr auf die Bekämpfung des ökonomischen Rückstands von Minderheitengruppen ausgerichtet war, als es dem Plan entsprach. 
Diese Verschiebung wurde noch verstärkt durch eine Empfehlung des renommierten wissenschaftlichen Rats für Regierungspolitik (Wetenschappelijke Raad voor her Regeringsbeleid). Mit seinem Bericht zur sogenannten „Allochtonen Politik" von 1989 empfahl der Rat der Regierung, sich auf die Bekämpfung sozio-ökonomischer Benachteiligung zu konzentrieren. Im Rahmen einer solchen Politik sollte „Kultur“ keinen Stellenwert mehr haben. Es gab jetzt andere Dringlichkeiten und der Rat war der Meinung, daß es von Einzelnen und Gruppen in der Gesellschaft abhinge, Werte und Normen zu bestimmen, an denen sie ihr Leben ausrichten wollten, und daß dieser Bereich nicht unter die Regierungsverantwortung fiele. Das hieß mit anderen Worten, die Regierung hat zwar kulturelle Verschiedenheit zu respektieren, ist aber nicht gehalten, sie aktiv zu fördern. Die Regierung übernahm diesen Standpunkt zur kulturellen Verschiedenheit. In einem späteren Stadium wurde diese Position weiter ausgefuihrt, insofern als die Regierung erklärte, ethnische Minderheiten hätten das Recht, im Einklang mit ihren kulturellen Werten und Normen zu leben, insoweit sich diese Werte mit den Grundprinzipien der niederländischen Verfassung vertrügen. ${ }^{3}$ Damit kam die holländische Minderheitenpolitik auf einen der Grundsätze liberaler öffentlicher Moral zurück: auf ethnische Neutralität. Diese Neutralität erfordert, daß der Staat es unterläßt, ein Urteil über die verschiedenen Konzeptionen guten Lebens abzugeben (d.h. die Werte und Normen, an denen Menschen ihr Leben ausrichten wollen, und daß es ihm nicht zusteht, irgendeine besondere Konzeption des Guten zu privilegieren (vgl. Kymlicka 1995a).

Die Auffassung kultureller Verschiedenheit, die sich in der Emanzipationspolitik niederschlug, war noch bis vor sehr kurzer Zeit weniger spektakulär. Die Eckwerte der Emanzipationspolitik sind Gleichberechtigung, Pluriformität (der Lebensstile) und Autonomie. In den als rechtsgültig anerkannten Schriftstücken zur Emanzipationspolitik, deren wichtigstes der Plan von 1985 ist, fehlen Bezüge auf Kultur nicht gänzlich, aber sie beziehen sich lediglich auf eine Kritik der Repräsentation von Geschlechterverhältnissen in der holländischen Kultur. Es wird anerkannt, daß es ein Machtgefälle zwischen den Geschlechtern gibt und daß es hinsichtlich verschiedener Bilder von Männlichkeit und Weiblichkeit einer kulturellen Veränderung bedarf, um dieses Machtgefälle aufzuheben. Sehr lange Zeit konzentrierte sich die Emanzipationspolitik auf die sozio-ökonomische Unabhängigkeit von Frauen und vernachlässigte dabei das Ziel der Pluriformität. So wurde die Emanzipationspolitik in erster Linie eine Politik zur Steigerung der Frauenerwerbsarbeit und der besseren Partizipation von Frauen auf dem Arbeitsmarkt. Frauen wurden also als eine Problemgruppe wahrgenommen, die einen Rückstand aufzuholen hatte, implizit wurde damit die Position von Männern zum Bezugspunkt gemacht und zur Norm erhoben (vgl. Bussemaker 1996).

Wir können abschließend feststellen, daß die Minderheitenpolitik mit einem deutlichen Anteil von Kulturpolitik begann, daß sie aber wie die Emanzipationspolitik als rein ökonomische Mangelpolitik endete. Damit soll nicht gesagt werden, daß Vorstellungen und Begriffe von Kultur bei der Festlegung der Politik gar keine Rolle spielen, sondern, daß sie gewissermaßen nur zum Subtext der politischen Strategien gehören. Ein Beispiel soll diesen Umstand illustrie- 
ren. Die holländische Regierung subventioniert im Rahmen ihrer Minderheitenpolitik bereits seit einigen Jahren ein großes Projekt für Familieninterventionsprogramme. Der den Programmen zugrundliegende Plan ist, da $B$ in den Augen holländischer Politiker die türkischen und marokkanischen Mütter durch ihre Erziehung die Kinder nicht angemessen auf die holländische Grundschule vorbereiten. Damit meint man (zum Teil) erklären zu können, warum ihre Kinder in der Schule versagen. Folglich müssen die Mütter lernen, ihre Kinder zu erziehen und infolgedessen gibt es einen Bedarf für Familieninterventionsprogramme. Da Kindererziehung in hohem Maße eine kulturelle Praxis ist, ist es klar, daß solche Programme eine direkte Intervention in die Kulturen ethnischer Minderheiten sind und daß, wenn sie erfolgreich sind, diese Programme zu einer Veränderung in den kulturellen Praktiken (bzw. kulturell determinierten Erziehungspraktiken) dieser Gemeinschaften führen werden. Die holländische Regierung betrachtet derartige Programme jedoch nicht als Kulturpolitik, sondern als Mangelkompensationspolitik. Kommen wir aber vom Subtext auf den Haupttext zurück: Was wird tatsächlich in den politischen Dokumenten über die Frauen ethnischer Minderheiten gesagt?

\section{Migrantinnen im Minderheitenmemorandum und im emanzipatorischen Plan}

Soweit es Frauen aus Minderheiten betrifft, folgt der emanzipatorischen Plan im großen und ganzen der mit dem Minderheitenmemorandum vorgelegten Analyse. Das Memorandum beschreibt die Situation von Frauen aus Minderheitsgruppen wie folgt:

Die meisten Frauen leben sozial isoliert. Es fehlt ihnen an hinreichenden Fähigkeiten und Kenntnissen, um in einer komplexen Industriegesellschaft unabhängig tätig sein zu können. Sie haben nur geringe oder gar keine Kenntnisse der holländischen Sprache und sie haben keine Chance, sich an der Gesellschaft zu orientieren, in der sie leben. Die vorhandenen Maßnahmen, mit denen beabsichtigt wurde, sie aus ihrer Isolierung herauszuholen, sie Holländisch zu lehren und sie mit der Gesellschaft vertraut zu machen, erreichen sie nicht in ausreichendem Maße (Minderheitenmemorandum 1983, 124).

Beiden Memoranden zufolge kommt die Mehrheit der Frauen aus Minderheitengruppen aus einer ländlichen Umgebung in ihrem Herkunftsland und richtet ihr Leben immer noch weitgehend an den traditionellen Werten und Normen aus (Minderheitenmemorandum 1983, 123). Hier liegt die Crux ihres Problems. Die anderen kulturellen Normen und Werte beinhalten eine andere Teilung von Aufgaben und Zuständigkeiten zwischen Männern und Frauen und dies ist der wesentliche Grund, warum es vielen dieser Frauen nicht gelingt, gesellschaftlich zu partizipieren.

Vor allem die Sätze über traditionelle Werte und Normen, die mangelnde Vertrautheit mit der holländischen Sprache und den Mangel an Kenntnissen 
und Fähigkeiten, deren es bedarf, um sich in der holländischen Gesellschaft selbst zu erhalten, lassen es als sehr wahrscheinlich vermuten, daB die oben erwähnten Abschnitte in erster Linie im Hinblick auf die Situation türkischer und marokkanischer Frauen geschrieben wurden. Von Frauen aus den früheren holländischen Kolonien (wie Surinam) wurde zur Zeit der Veröffentlichung der Dokumente angenommen, daß sie Holländisch sprächen und mit der holländischen Kultur vertraut seien. ${ }^{4}$ So zielten die politischen Dokumente also auf die türkischen und marokkanischen Frauen ab, die nicht imstande waren, sich in einer modernen Industriegesellschaft über Wasser zu halten.

Es ist außerdem bemerkenswert, daB in der Problemanalyse beider politischer Programme „traditionelle Werte und Normen“ mit einer , anderen Teilung von Aufgaben und Zuständigkeiten" verbunden werden, die dann wiederum mit der mangelnden Integration von Migrantinnen verknüpft werden. Es ließe sich fragen: Was für eine Tradition und wessen Tradition? Es macht den Anschein, als wäre hier mit vielen Worten (oder eher mit wenigen) eine Tradition umschrieben, in der der Mann als Ernährer gilt und die Frau als fürsorgliche Hausfrau, eine Tradition, die dem Mann ein Recht auf Leben in der Öffentlichkeit gibt, während die Frau dazu verurteilt ist, in der Privatheit des Hauses zu leben. Da es für niederländische Leser und Leserinnen offenkundig ist, daß man sich hierbei nicht auf surinamesische Traditionen bezieht, können diese ,traditionelle Werte und Normen" genauer als ,türkische und marokkanische" und also als ,islamische" Werte bestimmt werden. Daß diese Interpretation sich von der Substanz her als so naheliegend erweist, liegt an der niederländischen (populär-)wissenschaftlichen Literatur über marokkanische und türkische Immigranten, wenn diese Interpretation nicht sogar durch sie hervorgerufen wurde. In dieser Literatur werden nämlich „Islam“, „Tradition“ und „Frauendiskriminierung" in einem einzigen Deutungsmuster gebündelt, ohne jemals voneinander getrennt zu werden (vgl. Van der Zwaard 1995). Die Konsequenz daraus ist, daß sich im Minderheitenmemorandum nicht einmal das geringste Bedürfnis nach einer detaillierten Beschreibung von „Tradition“ erkennen läßt. ${ }^{5}$

Wenn wir den Problemaufriß über Frauen aus ethnischen Minderheiten mit dem über Minderheiten im allgemeinen vergleichen, so wird deutlich, daß ihre Position primär nach quantitativen Kategorien unterschieden wird: stärkere Rückständigkeit, größere Isolierung. Frauen aus Minderheitengruppen sind gewissermaßen mehr als zweifach in der Minderheit.

Im großen und ganzen folgt der emanzipationspolitische Plan der Problemanalyse des Minderheitenmemorandums. Es gibt jedoch einen signifikanten Unterschied. Im emanzipationspolitischen Plan wird anerkannt, daß das $\mathrm{Ge}$ schlechterproblem ein Problem ungleicher Machtverhältnisse ist. Deshalb führt der Plan den Begriff der doppelten Benachteiligung ein: Frauen aus Minderheitengruppen werden als Frauen benachteiligt und als Angehörige einer ethnischen Minorität. Wenn aber Geschlechterverhältnisse ein Gegenstand ungleicher Machtverhältnisse sind, dann stellt sich die Frage: Ist die Machtverteilung zwischen Männern und Frauen in ethnischen Minderheitengruppen im selben 
Ausma $\beta$ ungleich wie die Machtverteilung zwischen holländischen Männern und Frauen? Der Argumentationslinie des emanzipationspolitischen Plans folgend kann die Antwort nur negativ sein: Nein, die Kultur der ethnischen Minderheiten führt zu einer größeren Machtungleichheit. Allerdings läßt sich die Antwort nicht mit dem Kulturrelativismus in Einklang bringen, für den die holländische Regierung einsteht und demzufolge die Kultur ethnischer Minderheiten nicht unterlegen, sondern anders ist. Frauen aus Minderheitengruppen erfahren also doppelte Benachteiligung, aber sie sind nicht mehr unterdrückt und haben ein Recht auf Emanzipation ,unter Beachtung ihrer eigenen kulturellen Werte" (emanzipationspolitischer Plan 1985, 56). Deshalb wird der Vergleich mit holländischen Frauen, den der Begriff doppelte Benachteiligung nach sich zöge (doppelt im Vergleich mit wem?) nicht herausgearbeitet.

\section{Beschäftigung für türkische und marokkanische Frauen im Wohlfahrtsbereich}

Wenden wir uns von den Plänen weg und der Frage zu, wie sie in die Praxis umgesetzt wurden. Um die soziale Position von Migrantinnen zu verbessern, hatte die holländische Regierung ihre politischen Verfahrensrichtlinien zu Frauen und Minderheiten entwickelt. Für diese Politik gab es eine etwa zwölfjährige Erprobungsphase (1982-1994). Danach wurde diese politische Linie als separate aufgegeben und in die allgemeinen politischen Strategien zur Emanzipation und zu Minderheiten integriert. Ein wichtiger Bestandteil dieser allgemeinen politischen Strategie war ein Arbeitsbeschaffungsprojekt für türkische und marokkanische Frauen im Wohlfahrtsbereich. Es wurde in der zweiten Hälfte der achtziger Jahre durchgeführt.

Das Arbeitsbeschaffungsprojekt sollte etwa 100 Frauen umfassen. Es ging um Arbeit in der Familienhilfe und in sozialen Diensten und Sozialeinrichtungen. Die Form des Arbeitsbeschaffungsprojekts wurde gewählt, weil die Teilnahme von türkischen und marokkanischen Frauen am Arbeitsmarkt als schwierig eingeschätzt wurde. Ein weiteres Argument war, daß ihre Chancen, Zugang zu erlernten Berufen zu finden, sehr begrenzt seien. Um diese Chancen zu verbessern, wurde eine Kombination von Ausbildung und Erwerbsarbeit gewählt. Im Wohlfahrtsbereich standen solche Möglichkeiten auch Frauen mit geringen Bildungsvoraussetzungen offen. Ein weiterer Grund, in den Wohlfahrtsbereich hineinzugehen, war ,die Idee, daß eine höhere Repräsentanz von Mitarbeitern fremder Herkunft einen positiven Beitrag zur genaueren Abstimmung der sozialen Dienste auf die Bedürfnisse von Angehörigen ethnischer Minderheiten" leisten könnte (Abschlußbericht 1992/93, 24). Die Zielsetzung war übrigens nicht, eine Arbeitsteilung nach verschiedenen Klientenkategorien durchzusetzen. Mit Blick auf das politische Ziel der Integration sollten die Frauen sowohl mit holländischen wie mit fremden Klienten arbeiten.

Die Familienhilfe gibt Unterstützung im Haushalt oder bei der Pflege, manchmal wird dies durch persönliche Unterstützung oder Beratung ergänzt, 
vor allem in Fällen, wo Krankheit und Alter die Menschen daran hindern, sich ordentlich selbst zu versorgen. Es stellte sich jedoch heraus, daß türkische und marokkanische Frauen nicht viel Interesse an einer Arbeit in der Familienhilfe hatten. Die Erfahrungen von (zwölf) Frauen, die am Projekt teilnahmen, verdeutlichen, warum das so war.

Zunächst einmal konnten die Frauen innerhalb ihres Verwandten- und Bekanntenkreises nicht klar machen, worin ihre Arbeit bestand. In ihrer unmittelbaren Umgebung hielt man sie für, Putzfrauen', ein Beruf, der in der türkischen und marokkanischen Kultur den niedrigsten Status hat. Deshalb wollten die Frauen nicht in türkischen oder marokkanischen Familien eingesetzt werden. (...) Es ist immer noch so, daß die Frauen in ihrer unmittelbaren Umgebung nicht gerne über ihre Arbeit sprechen. Die Verständnislosigkeit gegen die sie oft anzukämpfen haben, birgt auch die Gefahr, Freunde zu verlieren. (...) Keine der Teilnehmerinnen hatte die Vorstellung, daß infolge ihrer Projektteilnahme mehr türkische oder marokkanische Familien leichteren Zugang zur Familienhilfe finden würden. (Projektbericht: Een koel hoofd en een warm hart, 33)

Diese Erfahrungen sind besonders beunruhigend, wenn berücksichtigt wird, welche Ziele mit dem Projekt erreicht werden sollten. Die Zielgruppe zeigte kein Interesse. Der Zugang zum Beruf kann ebenfalls nicht verbessert werden, da die Frauen über ihre Arbeit in ihrer eigenen Umgebung nicht sprechen können, und die Tatsache, daß sie in türkischen oder marokkanischen Familien nicht zum Einsatz kommen, bedeutet, daß es keine Nachfrage nach einer besseren Abstimmung der angebotenen Dienstleistungen gibt. Der Beruf setzt innerhalb ihres eigenen Umkreises einfach ihren Status herab, und das ist kaum emanzipatorisch. Darüber hinaus hebt die Arbeit auch die Isolierung der Frauen nicht auf, denn es ist eine sehr einsame Arbeit. Schließlich erweist sie sich als eine Arbeit, die die Frauen auch ohne irgendeine Qualifikation hätten ausführen können. Denn keineswegs alle Familienhilfeeinrichtungen legen Wert auf eine Ausbildung, landesweit liegt der Prozentsatz ausgebildeter Mitarbeiter zwischen 5 und 7. In der Alltagspraxis ist dies also ein ungelernter Beruf.

Der institutionalisierte Arbeitssektor deckt ein weites Feld ab, er reicht von der Arbeit in Krippen bis zur Arbeit in Frauenhäusern oder Obdachloseneinrichtungen. Die wichtigste Aufgabe von Sozialarbeit besteht darin, Menschen zu unterstützen, die in ihrer unmittelbaren persönlichen Situation in Schwierigkeiten geraten sind. Diese Unterstützung ist sowohl sozialpsychologischer wie praktischer Art. Auch in diesem Zusammenhang sind die Erfahrungen der Projektteilnehmerinnen nicht sehr positiv. Sie beklagen sich über Vorurteile und Diskriminierung in den Einrichtungen, für die sie arbeiten. In der Sozialarbeit werden sie zum Beispiel hauptsächlich für ihre eigene Zielgruppe eingesetzt, was in einer Reihe von Einrichtungen zwischen Teilnehmerinnen und Institution zu Konflikten geführt hat. Einer der Gründe dafür war, daß es hieß: „die Teilnehmerinnen sollten sich in der Praxis erst mit ihren eigenen Leuten befassen, 
bevor sie auf holländische Klienten losgelassen werden" (Projektbericht: De helpende hand 1988, 62). Es wurde auch die Chance einer verbesserten Professionalität in der Auseinandersetzung mit Klienten fremder Herkunft nicht wahrgenommen, die sich aus einer Einbeziehung der Projektteilnehmerinnen ergeben hätte. Trotz anderer Vereinbarungen fand schließlich nur ein Teil der Frauen nach $A b s c h l u ß$ des Projekts eine Arbeit und viele hatten das Gefühl, ausgenutzt worden zu sein. Der Projektbericht deutet an, daß viele Institutionen wohl nur deshalb teilgenommen hatten, weil das Projekt zeitweise einen Ausgleich für den Personalmangel bei Sozialarbeitern bot.

\section{Neue politische Entwicklungen}

Anhand von drei vor kurzem veröffentlichten Memoranden kann man sich ein Bild über die jüngsten Entwicklungen in der Politik machen. Es handelt sich um das Memorandum „Emanzipation in der Praxis“, mit dem der Kurs der Emanzipationspolitik nach 1995 festgelegt wird, um den Jahresbericht zur Minderheitenpolitik von 1997 und die Schrift zur Emanzipationspolitik von 1997 (Beleidsbrief Emancipatie). Die Emanzipationsmemoranden geben zu verstehen, daß die Emanzipationspolitik gegenwärtig einen Prozeß der Neuorientierung durchmacht. Beide wurden von der holländischen Regierung als Antwort auf die Kritik holländischer Frauenorganisationen und Migrantinnenorganisationen herausgebracht. Die Kritik bezog sich auf zwei Punkte. Erstens wurde vorgebracht, daß die Emanzipationspolitik ganze Bevölkerungsgruppen als Verlierer stigmatisiere, als ob Frau oder Migrantin zu sein, schon an sich ein Problem wäre, während das wirkliche Problem, wie nämlich die Gesellschaft strukturiert sei, außer Sichtweite bliebe. Der zweite Kritikpunkt war, $\mathrm{da} \beta$ das kulturelle Ziel der Emanzipationspolitik, Pluriformität, nicht in ausreichendem Maße verwirklicht worden sei (vgl. AISA 1996, Emancipatie Raad 1996). In den Memoranden wird diese Kritik aufgenommen und in eine Politik des „Mainstreaming“ eingearbeitet, die mit einer positiven Bewertung kultureller Verschiedenheit verbunden wird. „Mainstreaming“" von Emanzipation als öffentliches Interesse wurde als Formel von der vierten Weltfrauenkonferenz in Peking übernommen. Für die holländische Regierung bedeutet das, jetzt mehr Kooperation mit anderen sozialen Gruppierungen in der Gesellschaft zu suchen. Verbunden mit einer Neubewertung kultureller Verschiedenheit führt dies zu einer politischen Strategie, die sich nicht mehr auf die Bekämpfung von Deprivation ausrichtet und in der im großen und ganzen die Vorstellung aufgegeben wurde, es müsse für besondere Frauengruppen spezielle politische Maßnahmen geben. Statt dessen zielt die politische Strategie nun darauf ab, soziale Gruppierungen zu aktivieren und sie dazu anzuregen, in der Verschiedenheit eine „Quelle der Inspiration“ und der „Bereicherung“ zu erkennen. Das wichtigste politische Instrument zur Erlangung dieses Ziels ist „Kommunikation“. Und das unter diesem Vorzeichen wichtigste politische Projekt ist „Chancen im Betrieb“, eine Kampagne, die seit 1996 Unternehmen dazu animiert, die Begabungen von Frauen zu nutzen und auszuschöpfen, ins- 
besondere die sehr speziellen Begabungen von Migrantinnen (vgl. Beleidsbrief Emancipatie 5, 8-15). Trotz einiger Lippenbekenntnisse zum Paradigma der „Verschiedenheit" 6 ist die Minderheitenpolitik nach wie vor in hohem Maße darauf ausgerichtet, ökonomische Nachteile zu bekämpfen. So ist es nicht überraschend, daß die Minderheitenpolitik viele Maßnahmen vorsieht, mit denen die ökonomische Position von Migranten verbessert werden soll. Der Weg, den die Minderheitenpolitik einschlägt, verläuft anders als in der Emanzipationspolitik nicht über „,sanfte Überredung“, sondern erfordert den Einsatz härterer politischer Instrumente wie etwa Förderungsprogramme für ausgewählte Kategorien von Minderheiten. Im Minderheitenbericht von 1997 werden übrigens „Migrantinnen“ als ausgewählte Zielgruppe genannt, aber bis jetzt hat das noch nicht dazu geführt, daß entsprechende politische Maßnahmen ergriffen worden wären.

\section{Schluß}

Seit 1982 bildeten Frauen aus ethnischen Minderheiten in den Niederlanden eine gesonderte politische Kategorie. Für diese Zielgruppe wurde eine kombinierte Frauen- und Minderheitenpolitik entwickelt. Die Begründung für diese Politik ist im Minderheitenmemorandum von 1983 enthalten und im emanzipationspolitischen Plan von 1985.

Beide Memoranden behandeln das Problem von Frauen aus ethnischen Minderheitengruppen als ein Problem sozialer Benachteiligung. Die meisten Migrantinnen befinden sich diesen Memoranden zufolge in einer Situation sozialer Isolierung, hervorgerufen durch ihre Kultur, in diesem Fall ihre traditionellen Normen und Werte, und durch den Umstand, daß sie nicht über ausreichende Kenntnisse und Fertigkeiten verfügen, um sich unabhängig in der Gesellschaft durchsetzen zu können. Man ist der Meinung, daß sie als Frauen stärker in ihre ethnische Kultur eingebunden sind als ihre Männer und daher auch isolierter.

Einer der Punkte, die die Memoranden gemeinsam haben, ist, daß sie beanspruchen, die Situation aller Frauen aus ethnischen Minderheiten zu beschreiben, während sie tatsächlich nur auf türkische und marokkanische Frauen zutreffen. Die Frauendarstellung in diesen politischen Programmen entspricht in jeder Hinsicht den Stereotypen des Repräsentationssystems des Orientalismus. Die Frauen kommen aus unterentwickelten Gesellschaften, ihr Leben wird von Traditionen beherrscht, und die Diskriminierung, der sie ausgesetzt sind, wird als Konsequenz ihrer Unfähigkeit betrachtet, es mit der „normalen“ Routine des Alltagslebens in den Niederlanden aufzunehmen. Das Bild der unterentwickelten, unterdrückten östlichen Frau war offenbar so fest in die Netzhaut holländischer Politiker eingebrannt, daß sie vergessen konnten, daß die Mehrheit ihrer Zielgruppe aus dem Westen (Westindien) kam. Ein bedeutender Unterschied zwischen Minderheitenmemorandum und Emanzipationsmemorandum ist, daß letzteres den Zusatz macht, Frauen aus ethnischen Minderheiten seien in einer Situation doppelter Benachteiligung, als Frauen und als An- 
gehörige einer ethnischen Minderheit. Es erscheint dann so, als sei diese doppelte Benachteiligung durch die Normen und Werte hervorgerufen, die in der eigenen ethnischen Kultur hoch gehalten werden. So kommt die Spannung zwischen den freiheitlich-liberalen Werten, auf welche sich die Politik beruft, einerseits und der relativistischen Haltung andererseits deutlicher zum Vorschein als im Minderheitenmemorandum. Wenn vorausgesetzt wird, da $B$ alle Kulturen prinzipiell gleich sind, so kann nicht beansprucht werden, daß die eine Kultur (für Frauen) besser ist als die andere. Wenn aber dieser Schluß vermieden werden soll, so gibt es kaum eine andere Alternative als den Begriff „verschieden“ zu verwenden, wo ,unterlegen" oder ,ungleicher" gemeint ist. Genau das war es aber, was im emanzipationspolitischen Plan passierte.

Bei der Implementierung von politischen Programmen scheint der Kulturkonflikt in einer ziemlich naiven, wenn nicht nachlässigen Weise angepackt zu werden. Die Abneigung türkischer oder marokkanischer Familienhelferinnen gegen die Arbeit mit der eigenen Gruppe wird zwar respektiert, aber eine andere politische Linie wird nicht einmal erwogen. Besonders im Hinblick auf Emanzipation wäre es folgerichtig gewesen, wenn die holländische Regierung den betroffenen ethnischen Gruppen klargemacht hätte, daß sie Hausarbeit nicht als niedrige Arbeitstätigkeit ansieht. Auf der anderen Seite ist auch respektiert worden, daß die Institutionen ihrem eigenen kulturell determinierten Prozedere stark verhaftet blieben. Auch hier hätte es zum Beispiel eine politische Option geben können, die Kooperation zu beenden, wenn die entsprechenden Einrichtungen sich weiterhin weigerten, ihre Verfahrensweisen an die kulturelle Verschiedenheit ihrer Klientel anzupassen.

Anfänglich zeigte die holländische Minderheitenpolitik eine gewisse Sensibilität für Belange kultureller Verschiedenheit, diese wurde aber schnell aufgegeben zugunsten einer Politik, die darauf abzielte, die ökonomischen und sozialen Nachteile gesellschaftlicher Gruppen zu bekämpfen. Im Rahmen dieser Politik, die Minderheitengruppen als Problemgruppen betrachtet, erscheint kulturelle Differenz als ein gesellschaftliches Handicap, das überwunden werden muß. Im besten Fall läßt diese Perspektive Raum für die Toleranz von Pluriformität, gruppenspezifische kulturelle Differenz, aber das gilt nicht als etwas Erstrebenswertes.

Die heutige Emanzipationspolitik scheint einen Bruch mit dieser Tradition zu vollziehen, insofern als sie behauptet, kulturelle Differenz hoch zu schätzen. Diese Feier der Differenz läßt jedoch wegen ihrer unkritischen Akzeptanz von Minderheitenkulturen ebenso wenig Raum für eine kritische Reflexion des Zusammenhangs von Werteunterschieden und Frauenemanzipation.

Zusammenfassend läßt sich sagen: Weder in der Theorie noch in der Praxis scheint die holländische Politik eine angemessene Antwort auf die Frage gefunden zu haben, ob unterschiedliche Kulturen gleich behandelt werden sollten oder nicht. Unklar ist auch geblieben, wie die Werte der Minderheitenpolitik und Emanzipation miteinander zu verbinden sind und wie individuelle Rechte sich zu Gruppenrechten und -politik verhalten.

Meine Sympathien gelten einer Gesellschaft, welche die öffentliche Manifestation von Gruppendifferenzen und -identitäten nicht nur toleriert, sondern 
aktiv fördert. Aber es ist wohl vorhersehbar, daß die Kulturen dann miteinander in Konflikt geraten. Gerade weil aber viele interkulturelle Wertekonflikte das Leben der Frauen betreffen, möchte ich mich für die Ausformulierung einer regierungspolitischen Strategie einsetzen, welche die kulturelle Verschiedenheit berücksichtigt, ohne die Rechte und Interessen von Frauen aus dem Blick zu verlieren.

\section{Anmerkungen}

1 Vgl. zum Beispiel Bader 1995, Goldberg 1994, Gutmann 1992, Kymlicka 1995a, 1995b, Miller und Walzer 1995, Seidmann 1995.

2 Eine Anmerkung zur Terminologie: Der in der holländischen Politik verwendete offizielle Begriff ist „ethnische Minderheiten“, häufig abgeküzt mit „Minderheiten“. Ich werde sie abwechselnd als solche oder als Migranten bezeichnen und, wo es von Bedeutung ist, als „kulturelle" Minderheiten.

3 Diese Formulierung war das Ergebnis einer breiten öffentlichen Diskussion über Multikulturalismus im Jahre 1991. Die Innenministerin, die für Minderheitenfragen die unmittelbar zuständige Ministerin ist, sagte in ihrer Antwort auf diese Debatte, daB „Minderheiten begreifen müssen, daß eine Reihe von Grundwerten der niederländischen Gesellschaft nicht zur Verhandlung stehen". Darunter individuelle Autonomie und Gleichheit von Mann und Frau. (Het maatschapelijk debat integratie. Brief van de minister van binnenlandse zaken, 27. März 1992)

4 In Wirklichkeit haben sie Probleme mit der holländischen Sprache, aber das wurde erst viel später entdeckt.

5 Das hat sich im Laufe der Zeit nicht geändert. Im Januar 1997 gab es zum Beispiel einen Medienrummel wegen einer Äußerung der Gesundheitsministerin. Die Ministerin sagte, sie könne sich vorstellen, daß eine Frau aus einer fremden Kultur sich für eine Abtreibung entschiede, wenn der Fötus weiblich sei. Alles Diskutanten hatten ,fremde Kultur" als „muslimische Kultur" verstanden.

6 Im Minderheitenbericht von 1997 liest man gelegentlich Sätze wie: „Die Gesellschaft sollte das ökonomische, soziale und kulturelle Potential von Minderheiten nutzen." (S. 7)

\section{Literatur}

Een koel hoofd en een warm hart. Verslag van een onderzoek naar de opleiding en het functioneren vah Turkse en Marokkaanse gezinshelpenden. (1988) Den Haag: Ministerie van Sociale Zaken en Werkgelegenheid.

Ahmed, Leila (1982): , Western ethnocentrism and perceptions of the harem'. Feminist Studies 8/3, pp. 521-554.

Ahmed, Leila (1992): Women and Gender in Islam. Historical Roots of a Modern Debate. New Haven/London.

Allochtonen Beleid (1989), Advies van de Wetenschappelijke Raad voor het Regeringsbeleid, Den Haag.

AISA (1996): Met kracht naar empowerment: empowerment-visie van zwarte, migranten-en vluchtelingenvrouwen. Utrecht: Project AISA.

Bussemaker, Jet (ed.) (1996): Struikelblokken en verbindingswegen. nieuwe oriëntaties voor het emancipatiebeleid. Den Haag.

Beleidsplan Emancipatie (1985), 2e Kamer, 1984-1985, 19052, 1-2. 
Bader, Veit M. (1995): Rassismus, Ethnizität, Bürgerschaft. Soziologische und philosophische Überlegungen. Münster.

Emancipatie in uitvoering. Koersbepaling van het emancipatiebeleid na 1995. 2e Kamer, 1995-1996, 24406, 4.

Emancipatie Raad (1996): Aankoersen op een geëmancipeerde samenleving. Den Haag.

Final Report Women and Minority Policy. Eindrapportage Vrouwen en Minderhedenbeleid (1992), Den Haag: Ministrie van Sociale Zaken en Werkgelegenheid.

Gutmann, Amy (ed.) (1992): Multiculturalism and the Politics of Difference. Princeton.

Goldberg, Daniel T. (ed.) (1994): Multiculturalism. A Critical Reader. Oxford UK \& Cambridge USA.

Kabbani, Rana (1986): Europe's Myth of Orient. Devise and Rule. London.

Kymlicka, Will (1995a): Multicultural Citizenship: A Liberal Theory of Minority Rights. Oxford.

Kymlicka, Will (ed.) (1995b): The rights of Minority Cultures. Oxford.

Lewis, Ruth (ed.) (1996): Gendering Orientalism. Race, Femininity and Representation. London/New York.

Lutz, Helma (1991): Migrant Women of ,Islamic Background'. Images and Selfimages. Amsterdam, Middle East Research Associates: Occasional Paper no. 11.

Miller, David/Walzer, Michael (eds.) (1995): Pluralism, Justice and Equality. Oxford.

Minderhedennota, 2e Kamer, 1982-1983, 19102, 20-21.

Beleidsbrief Emancipatiebeleid 1997. (1996) Den Haag: Ministerie van Sociale Zaken en Werkangelegenheid.

Minderhedenbeleid 1997. Jaaroverzicht Integratiebeleid Etnische Minderheden. Den Haag. 2e Kamer, 1996-1997, 24401, $1 / 2$.

De helpende hand: verslag van een onderzoek naar de opleiding en het functioneren van Turkse en Marokkaanse hulpverleensters. (1988) Den Haag: Ministerie van Sociale Zaken en Werkgelegenheid.

, Het maatschappelijk debat integratie. Brief van de Minister van Binnenlandse Zaken : Maart 271992.

Saadawi, Nawal El (1980): The Hidden Face of Eve. Women in the Arab World. London.

Said, Edward W. (1978): Orientalism. London.

Seidman, Steven (1995): ,Difference and Democracy in Western Nations: Some Conceptual and Comparative Observations.' Lecture given in Amsterdam, published in Krisis 60 . pp. 60-74.

Zwaard, Joke van der (1995): ,Naughty boys and Obstinate Girls. District Nurses ' Cultural and Professional Explanations of Child Rearing Practices'. In: Inge Boer, Annelies Moors \& Toon van Teeffelen (eds.): Orientations. Changing stories; Postmodernism and the ArabIslamic World, Amsterdam/Atlanta, Rodopi, pp. 133-145. 